\title{
A micro-CT study of microstructure change of alveolar bone during orthodontic tooth movement under different force magnitudes in rats
}

\author{
JINGTAO AN ${ }^{1}$, YING LI $^{2}$, ZHONGSHUANG LIU ${ }^{2}$, RUI WANG ${ }^{3}$ and BIN ZHANG ${ }^{2,4}$ \\ ${ }^{1}$ Department of Orthodontics, School of Stomatology, Harbin Medical University, Harbin, Heilongjiang 150001; \\ ${ }^{2}$ Institute of Hard Tissue Development and Regeneration, The Second Affiliated Hospital of Harbin Medical University, \\ Harbin, Heilongjiang 150081; ${ }^{3}$ Department of Pediatrics, School of Stomatology, Harbin Medical University, Harbin, \\ Heilongjiang 150001; ${ }^{4}$ Heilongjiang Academy of Medical Sciences, Harbin, Heilongjiang 150086, P.R. China
}

Received July 29, 2016; Accepted February 2, 2017

DOI: 10.3892/etm.2017.4186

\begin{abstract}
The dynamic changes of the microstructure of alveolar bone during orthodontic tooth movement in rats was explored by employing micro-computed tomography (micro-CT) system and to provide theoretical reference for clinical orthodontic treatment. Ten rats were selected randomly as control among 70 adult female Wistar rats, and the other 60 rats were divided into $25-\mathrm{g}$ and 75 -g groups of equal number. Orthodontic appliance with force of $25 \mathrm{~g}$ and $75 \mathrm{~g}$ was installed to perform the molar mesial movement. Microstructural parameters for trabecular bone mesial to the distobuccal root were evaluated at different time points using micro-CT system. Moreover, distance for mesial movement of the molar were measured. Microstructural parameters for trabecular bone of two groups showed no significant changes from day 0 to day 3 ( $\mathrm{P}>0.05$ ); from day 3 to day 7, bone mineral density (BMD), bone volume/total volume (BV/TV) and trabecular thickness (Tb.Th) decreased significantly $(\mathrm{P}<0.05)$, whereas trabecular separation (Tb.Sp) and structure model index (SMI) increased significantly $(\mathrm{P}<0.05)$; from day 7 to day 14 , in 25 -g group, BMD, BV/TV and Tb.Th increased significantly $(\mathrm{P}<0.05)$, while Tb.Sp and SMI decreased significantly $(\mathrm{P}<0.05)$. Correspondingly, in 75-g group, changes of parameters did not carry any statistical significance $(\mathrm{P}>0.05)$. Furthermore, the 75-g group showed larger distance than 25-g group only at day $14(\mathrm{P}<0.05)$. In conclusion, in order to maintain the health of periodontal tissues, adequate time for repair and recovery is needed to ensure reasonable remolding of alveolar bone and healthy movement of the orthodontic tooth.
\end{abstract}

Correspondence to: Dr Bin Zhang, Institute of Hard Tissue Development and Regeneration, The Second Affiliated Hospital of Harbin Medical University, 246 Xue Fu Road, Harbin, Heilongjiang 150081, P.R. China

E-mail: zhangbinyzz@126.com

Key words: micro-CT, microstructure, alveolar bone, orthodontic tooth movement, trabecular bone

\section{Introduction}

During the process of orthodontic treatment, tooth movement is closely related to alveolar bone remodeling, which consists of bone resorption in direction of tooth movement and bone formation on the opposite side. The use of an optimal force system is important for an adequate biological response in the periodontal system. Heavy forces could overcompress the periodontal ligament and induce hyalinization, which could impede resorption of the alveolar bone surface $(1,2)$.

A previous study on the periodontal tissues, and especially on the microstructure of the trabecular bone used to adopt specimens destructive methods of histological techniques which was capable of the two-dimensional microstructure, but was hard to obtain and describe the parameters of the alveolar bone internal microstructure (3). Micro-computed tomography (micro-CT) is a new imaging examination and analysis technique of high resolution and non-intrusive has developed fast in recent years, which can provide three-dimensional qualitative as well as quantitative data of the tested specimens, to better understand and study the remolding of alveolar bone microstructure $(4,5)$.

The purpose of this study was to evaluate the dynamic changes of the microstructure of alveolar bone during orthodontic tooth movement under different force magnitudes in rats employing micro-CT system, and to explore the pattern of changes of the trabecular bone microstructure in the alveolar bone so as to provide theoretical reference for clinical orthodontic treatment.

\section{Materials and methods}

Grouping of the laboratory animals. Seventy 11-week-old adult female Wistar rats (approximate weight, 220-260 g) were used for this study. A total of 10 rats were selected randomly as control, and the remaining 60 rats were divided into $25-\mathrm{g}$ and $75-\mathrm{g}$ groups of equal number. The rats were raised under standard conditions: Room temperature at $25 \pm 1^{\circ} \mathrm{C}$, relative humidity at $55 \pm 5 \%$, and 12 -h light and dark cycles. All the experimental protocols followed were approved by the Ethics 
Committee of Harbin Medical University, and the experiments were carried out under the control of the University's Guidelines for Animal Experimentation.

Installing orthodontic appliance. A total of 30 rats of 25 -g group and 30 rats of $75-\mathrm{g}$ group were anesthetized by intraperitoneal injection of $10 \%$ chloral hydrate, a cervical groove was prepared on the incisors using a round burr with a dental low-speed handpiece (NSK Ltd., Tokyo, Japan). The maxillary left first molar was connected with both maxillary central incisors with nickel-titanium coil spring 0.008 inch in diameter (Shinye Odontology Materials Corp. Co., Hangzhou, China) using a $0.2 \mathrm{~mm}$ ligature wire (Changsha Tiantian Dental Equipment Co., Ltd., Changsha, China) to perform the mesial movement (Fig. 1). The ligature wire was then secured with bond adhesive (Transbond; 3M Unitek, Monrovia, CA, USA) on the incisors. Forces of 25 and $75 \mathrm{~g}$ were applied separately to observe the reactions of the alveolar bone. Spring retention was checked daily to ensure the stability of the applied force. The incisors, molar, and spring were cleaned and irrigated with tap water as needed to prevent potential trauma and irritation to the gingival and periodontal tissues.

Experimental samples. A total of 10 rats as control without installing orthodontic appliance were euthanized by perfusion though heart with $4 \%$ paraformaldehyde on day 0 , and every 10 rats in each group were euthanized by the same way on days 3, 7 and 14, respectively. Then the maxillae were dissected and stored in $4 \%$ paraformaldehyde.

Micro-CT scanning. A total of 70 samples were scanned with a micro-CT scanner ( $\mu$ CT 35; Scanco Medical AG, Bassersdorf, Switzerland) with a $7 \mu \mathrm{m}$ voxel size using the following parameters: $114 \mathrm{~mA}, 70 \mathrm{kVp}$, exposure time of $300 \mathrm{msec}$, and a horizontal scan angle. The scanning procedure lasted $\sim 1 \mathrm{~h}$ per sample and generated $\sim 1,000$ two-dimensional images with a resolution of $1,024 \times 1,024$ pixels.

Evaluating the microstructural parameters. The region of interest (ROI) of the alveolar bone was selected according to previously described method which showed the integral microstructure of alveolar bone (6). A 210x210x210 $\mu \mathrm{m}$ cube of trabecular bone mesial to the cervial third of the distobuccal root of the maxillary left first molar was selected separately for analysis. The distance between the cube and the root was $100 \mu \mathrm{m}$ (Fig. 2).

Three-dimensional microstructure of the alveolar bone was analyzed using the software affiliated to the micro-CT (Scanco ${ }^{\circledR}$ software ver. 3.7; Scanco Medical $\mathrm{AG}^{\circledR}$, Brüttisellen, Switzerland). We evaluated the bone mineral density (BMD, $\mathrm{mg} \mathrm{cm}{ }^{-3}$ ); bone volume/total volume (BV/TV, \%); trabecular thickness (Tb.Th, mm); trabecular number (Tb.N, $\mathrm{mm}^{-1}$ ); and trabecular separation (Tb.Sp, $\mathrm{mm}$ ), microstructure model index (SMI), showing whether the trabecular bone is microstructured as rod-like or plate-like. Theoretical value for a complete rod-like microstructure is 3 , while theoretical value for an ideal plate-like microstructure is 0 . The stress that the tabular microstructure can bear is larger than that of clave microstructure $(6,7)$.

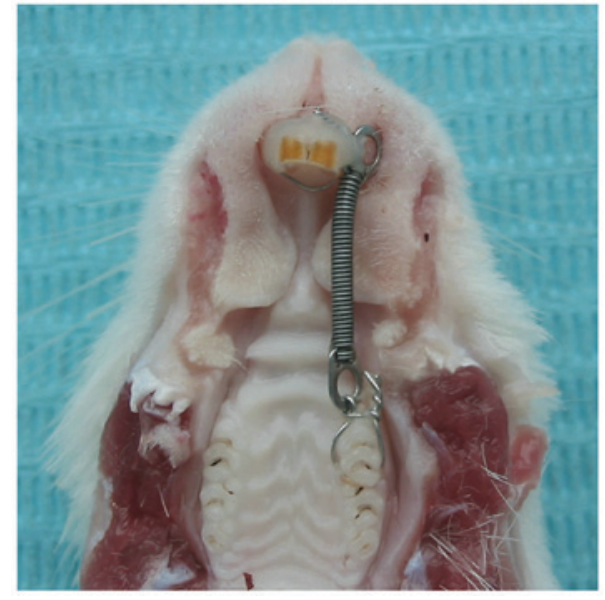

Figure 1. The orthodontic appliance is installed to the maxillary left first molar and the incisors.
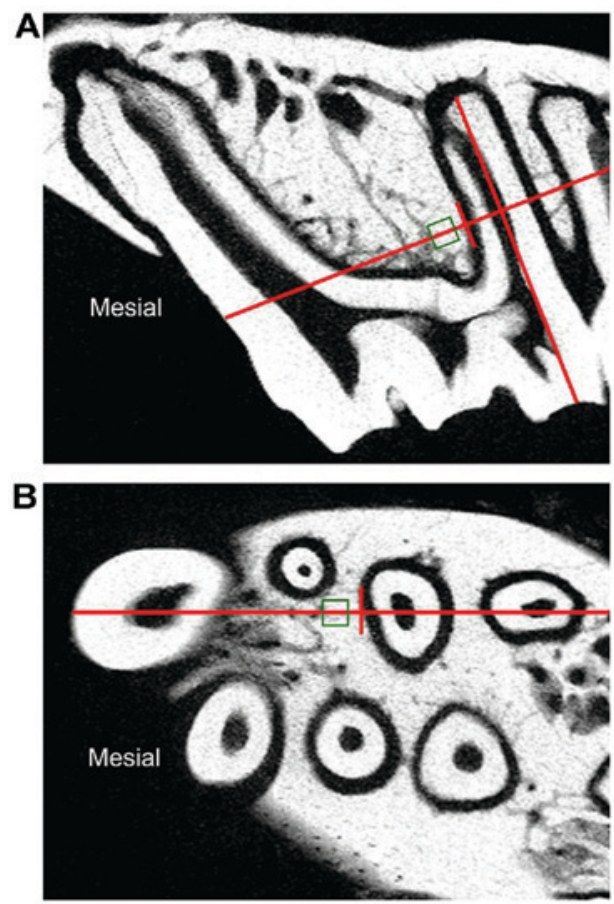

Figure 2. The region of interest. A cube of trabecular bone $(210 \times 210 \times 210 \mu \mathrm{m})$ mesial to the cervical third of the distobuccal root of the maxillary left first molar $(100 \mu \mathrm{m})$ was selected for analysis in micro-computed tomography images: (A) Sagittal surface passing the centers of mesial root and distobuccal root; (B) horizontal surface perpendicular to the cervical third of the distobuccal root.

Measuring the distance for mesial movement. Distance for mesial movement of the molar were measured between the nearest two landmark points $(a, b)$ in the crowns of the first molar (M1) and second molar (M2) which were indentified on the longitudinal section through maximal diameter of the distobuccal root and mesial root of the first molar (Fig. 3).

Statistical analysis. The data were analyzed by using SPSS, version 21.0 (SPSS Inc., Chicago, IL, USA). Parameters of 25 -g and 75-g groups on days $0,3,7$ and 14 were compared using Student-Newman-Keuls of ANOVA. P $<0.05$ was considered to indicate a statistically significant difference. 

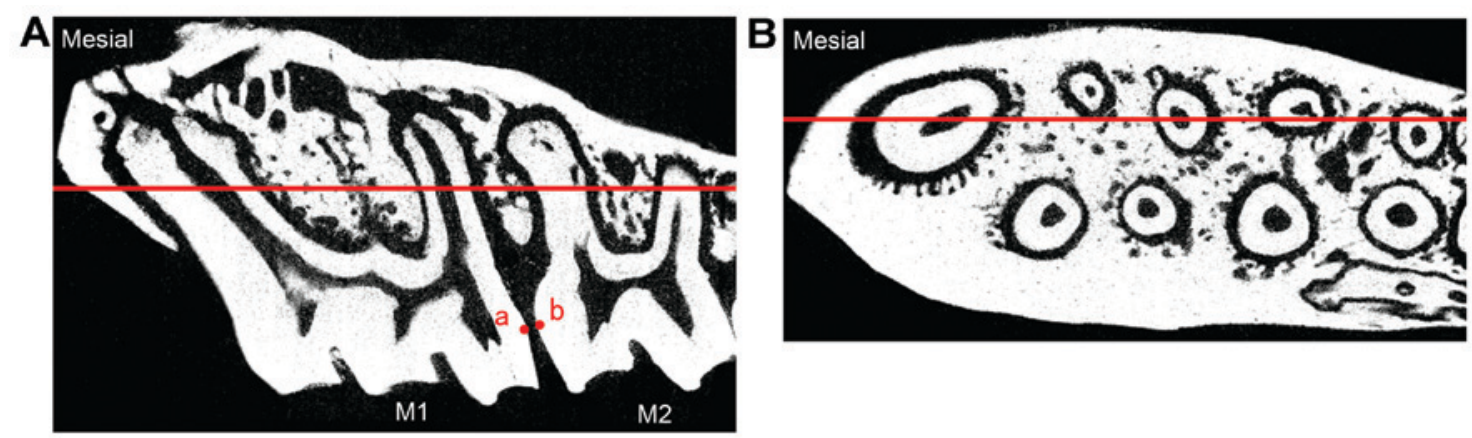

Figure 3. Landmark point locations and methods for the measurement of molar mesial movement in micro-computed tomography images. Landmark points a,b are indicated. M1, first molar; M2, second molar. (A) Sagittal view; (B) horizontal view.

A

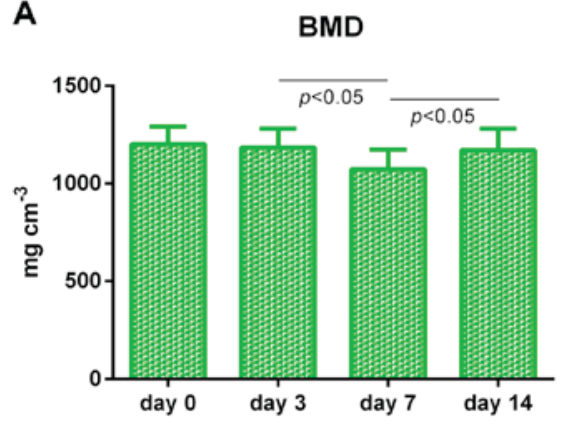

C

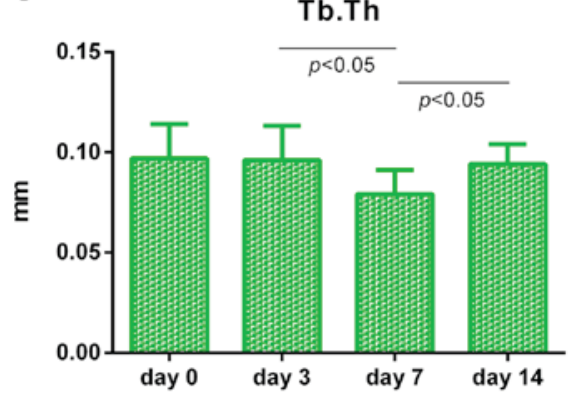

E

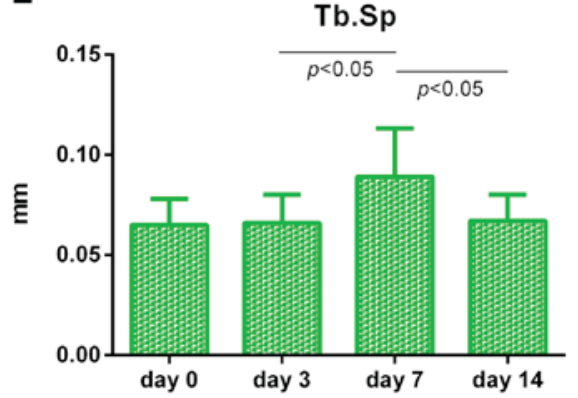

B

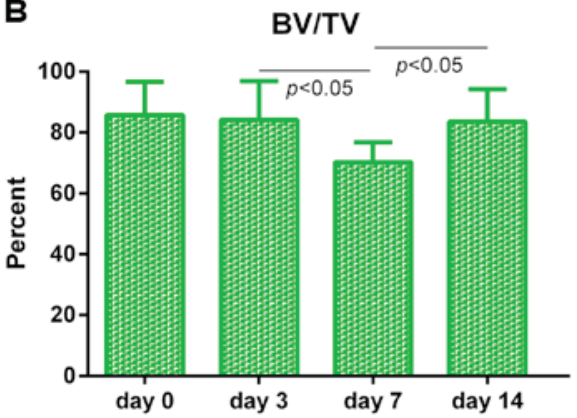

D

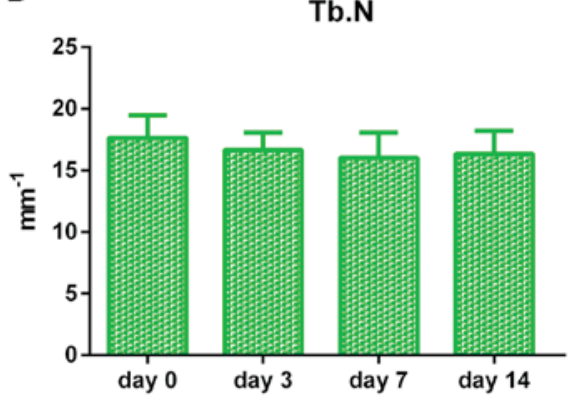

$\mathbf{F}$

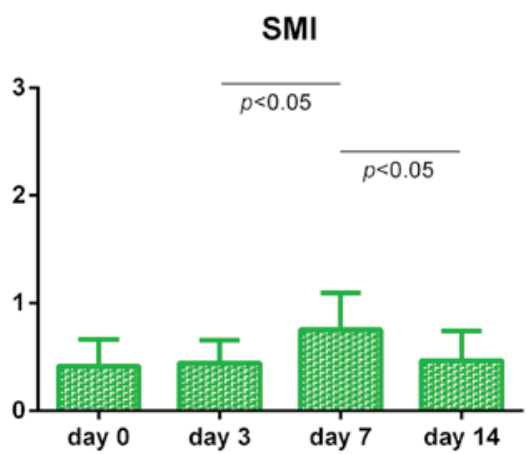

Figure 4. Microstructural parameters for trabecular bone mesial to the distobuccal root of the maxillary left first molar at different time points in 25-g group after installing orthodontic appliance. (A) Bone mineral density (BMD); (B) bone volume fraction (BV/TV); (C) trabecular thickness (Tb.Th); (D) trabecular number (Tb.N); (E) trabecular separation (Tb.Sp); (F) structure model index (SMI). Error bars indicate standard deviations.

\section{Results}

Microstructural parameters for trabecular bone mesial to the distobuccal root of the maxillary left first molar of 25-g group at different time-points are summarized in Fig. 4. From day 0 to day 3 , the parameters did not display any significant changes ( $\mathrm{P}>0.05$ ); from day 3 to day 7, BMD, BV/TV and Tb.Th significantly decreased $(\mathrm{P}<0.05)$, whereas Tb.Sp and SMI significantly increased $(\mathrm{P}<0.05)$; while from day 7 to day 14 , $\mathrm{BMD}, \mathrm{BV} / \mathrm{TV}$ and $\mathrm{Tb} . \mathrm{Th}$ increased significantly $(\mathrm{P}<0.05)$, 
A

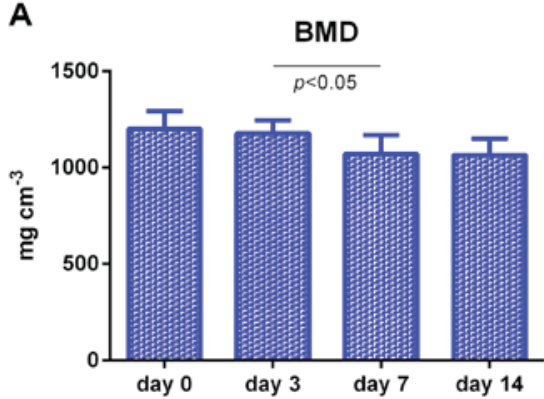

C

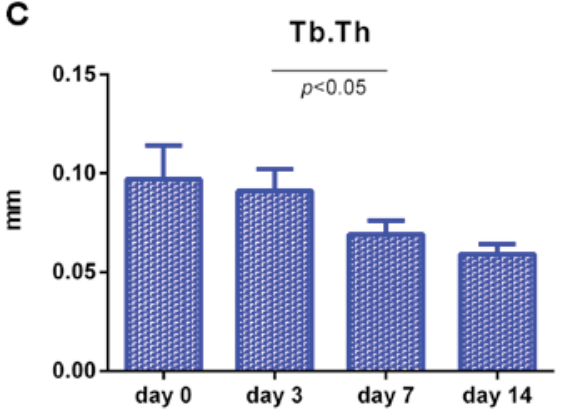

E

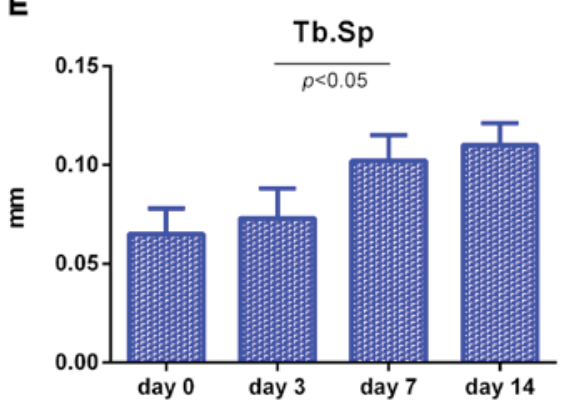

B

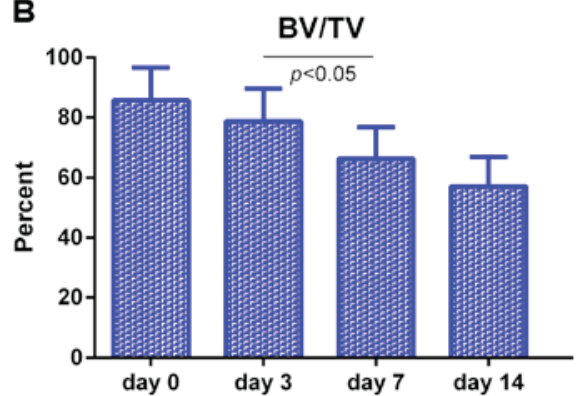

D

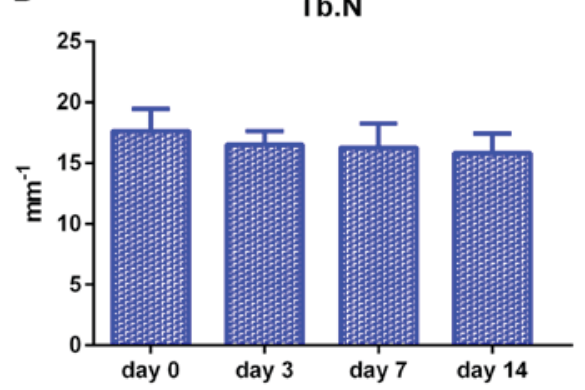

$\mathbf{F}$

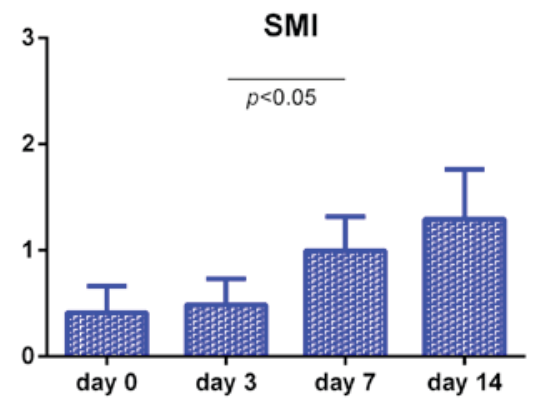

Figure 5. Microstructural parameters for trabecular bone mesial to the distobuccal root of the maxillary left first molar at different time points in 75-g group after installing orthodontic appliance. (A) Bone mineral density (BMD); (B) bone volume fraction (BV/TV); (C) trabecular thickness (Tb.Th); (D) trabecular number (Tb.N); (E) trabecular separation (Tb.Sp); (F) structure model index (SMI). Error bars indicate standard deviations.

while Tb.Sp and SMI decreased significantly $(\mathrm{P}<0.05)$. Tb.N did not have any clear change during the whole process of the experiment $(\mathrm{P}>0.05)$.

Microstructural parameters for trabecular bone mesial to the distobuccal root of the maxillary left first molar of 75-g group at different time points are summarized in Fig. 5. Statistical results of parameters on day 0 to day 7 are consistent with those of $25-\mathrm{g}$ group, while from day 7 to day 14 , changes of parameters did not carry any statistical significance ( $\mathrm{P}>0.05)$, Tb.N did not have any significant change during the whole process of the experiment $(\mathrm{P}>0.05)$.

Microstructural parameters for trabecular bone mesial to the distobuccal root of the maxillary left first molar between 25-g and 75-g groups at different scanning time points are summarized in Fig. 6. From day 0 to day 7, the change trends of microstructural parameters of two groups were similar, but from day 7 to day 14, the change in trends between two groups showed significant difference $(\mathrm{P}<0.05)$.

Distance for mesial movement of the molar at different scanning time points between $25-\mathrm{g}$ and $75-\mathrm{g}$ groups are summarized in Fig. 7. The 75-g rats showed larger distance than 25 -g only at day $14(\mathrm{P}<0.05)$.

\section{Discussion}

Previous studies frequently employed methods such as histological analysis to explore microstructure of the alveolar bone, which was sophisticated in operation and hard to obtain and describe the internal parameters of the specimens (3-5). The method of histological sections was also disadvantageous in limited number of slices and in turn deviation and substantial loss of information (3). In contrast, micro-CT is easy to operate with a high resolution of $\mu \mathrm{m}$ that is capable of accurate qualitative as well as quantitative analysis of the specimens. It allows improved study of the bone remolding and later histological or electron microscopic observation of the specimens, whenever needed, after having completed scanning $(4,5)$.

The model of mesial movement of the maxillary left first molar by means of orthodontic extrusion was first proposed by Waldo and Rothblatt (8). Based on literature review, Ren et al (9) held the view that one orthodontic force loading cycle should be 14 days. Repair and reconstruction of alveolar bone would be dominant after 14 days. Since the molar volume of human is 50 times than that of the rat, orthodontic extrusion 
A

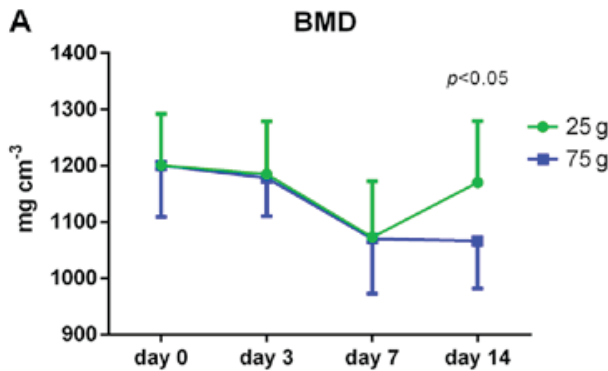

C

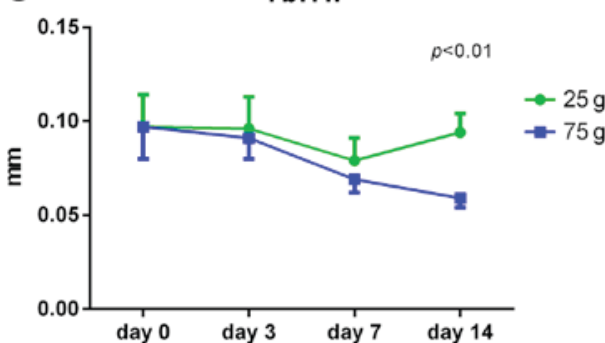

E

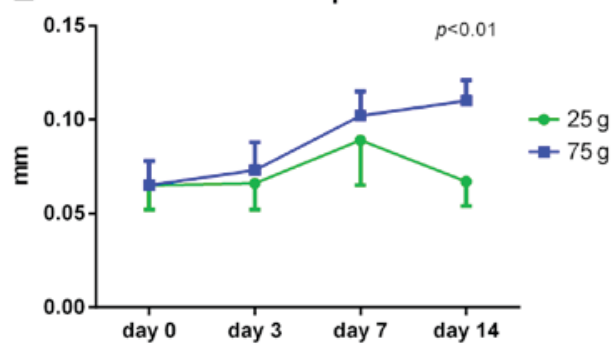

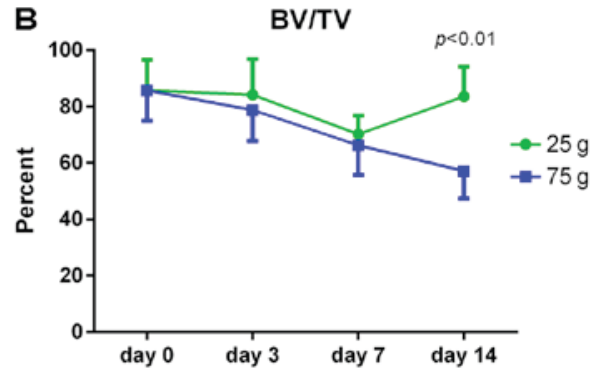

D

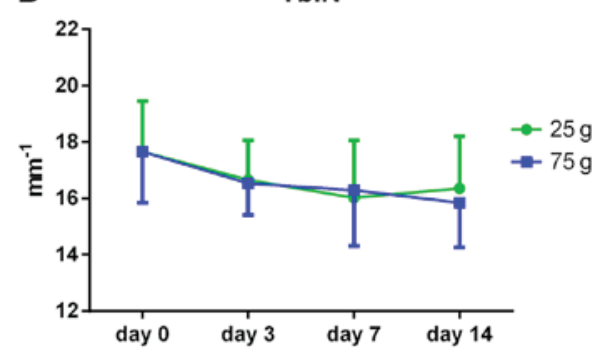

$\mathbf{F}$

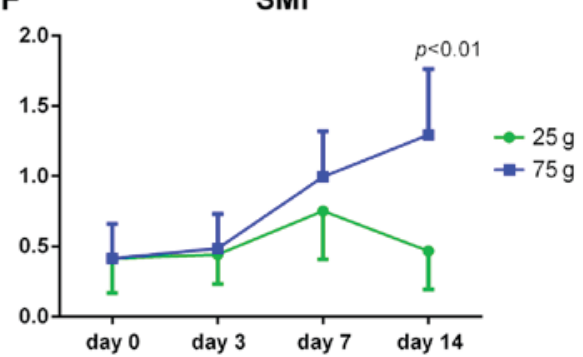

Figure 6. Microstructural parameters for trabecular bone mesial to the distobuccal root of the maxillary left first molar at different time points between 25-g and 75-g groups after installing orthodontic appliance. (A) Bone mineral density (BMD); (B) bone volume fraction (BV/TV); (C) trabecular thickness (Tb.Th); (D) trabecular number (Tb.N); (E) trabecular separation (Tb.Sp); (F) structure model index (SMI). Error bars indicate standard deviations.

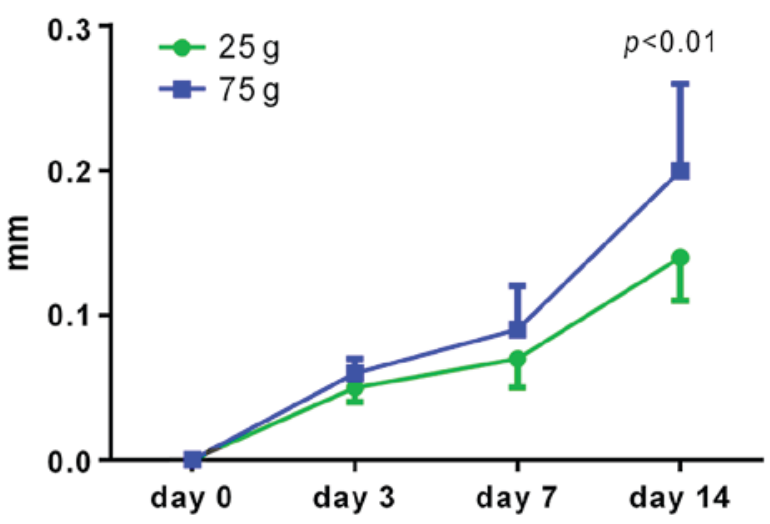

Figure 7. Distance for mesial movement of the maxillary left first molar at different time points between 25 -g and 75 -g groups after installing orthodontic appliance. Error bars indicate standard deviations.

of $20 \mathrm{~g}$ was considered as an ideal value for orthodontic molar tooth movement mesially, and $75 \mathrm{~g}$ belonged to heavy force. Taking the factor of the basic scale value of $25 \mathrm{~g}$ on the dynamometer used in the experiment, for more accurate measurement, this study selected 25 and $75 \mathrm{~g}$ orthodontic extrusion as the orthodontic force loading.
Based on the previous study on the three-dimensional finite elements of the rat molar, under the application of mesial force loading, the mesial side of the disbuccal root was subject to the largest compression (10). During the process of orthodontic tooth movement, alveolar bone on the compression side would pose the main resistance to the tooth movement, so if the alveolar bone on the compression side could always maintain a reasonable and stable rate of resorption and reconstruction throughout the orthodontic treatment, then the tooth would be able to move into the ideal position healthily and effectively. This study chose the alveolar bone mesial to the cervial third of the distobuccal root of maxillary first molar as its interest observation region, which is the location of the largest compression and of typical significance in terms of bone resorption and remodeling. Some studies using micro-CT system observed the changes of the microstructure of alveolar bone during orthodontic movement in vivo $(11,12)$. However, they did not choose the location of the largest compression as interest observation region, so the results of the experiments were affected inevitably. Moreover, our study which used 70 rats tried to avoid the result error caused by sample difference as far as possible.

Currently, there exists controversy from histological experimental studies concerning the time points of alveolar 
bone reconstruction during orthodontic tooth movement. Kohno et al (13) showed that hyaline change in the alveolar bone appeared on day 7 , and bone resorption and reconstruction took place on day 14 after the force loading. However, Tomizuka et al (14) indicated that bone resorption happened on day 10 after force loading through histological observation.

The results showed that on day 0 to day 3 after the application of the force loading, the parameters of the two groups did not have any evident change $(\mathrm{P}>0.05)$, indicating that resorption did not occur in the alveolar bone. From day 3 to day 7, BMD, BV/TV and Tb.Th significantly decreased $(\mathrm{P}<0.05)$, while Tb.Sp and SMI significantly increased $(\mathrm{P}<0.05)$, indicating that resorption occurred in the alveolar bone. The difference was that from day 7 to day 14 , in 25 -g group, BMD, BV/TV and Tb.Th increased significantly $(\mathrm{P}<0.05)$, while Tb.Sp and SMI decreased significantly $(\mathrm{P}<0.05)$, indicating that alveolar bone was in the phase of repair and reconstruction.

Correspondingly, in $75-\mathrm{g}$ group, changes of various parameters did not carry any statistical significance $(\mathrm{P}>0.05)$, indicating that alveolar bone was still in the phase of resorption. The comparison of microstructural parameters for trabecular bone between $25-\mathrm{g}$ and $75-\mathrm{g}$ groups at different scanning time points also supported above conclusion.

Obviously, on day 14, for the rats of 25-g group that were already in the repair and reconstruction, it was feasible to continue applying force loading; however, for the rats of $75-\mathrm{g}$ group that were still in the phase of resorption, it was disputable to continue applying any force loading. Thus, further studies are still needed.

Previous studies showed that orthodontic tooth movement included three phases: The first phase is initial rapid tooth movement after the force loading; the second phase is delayed tooth movement; the third phase is linear tooth movement (2,15-17). In our study, the tooth movement of both groups conformed with the description above. Among them, from day 0 to day 3 belonged to phase 1; from day 3 to day 7 was in phase 2; and from day 7 to day 14 belonged to phase 3 . However, 75-g group showed larger distance than 25-g group only at day $14(\mathrm{P}<0.05)$. Apparently, this kind of accelerated tooth movement at the expense of excessive alveolar bone was not ideal and healthy pattern.

The aforementioned findings may provide reference to orthodontic practitioners that, in order to maintain the health of periodontal tissues, low force should be advocated, and it is not suggestive to frequently apply orthodontic force loading. Periodontal tissues should be allowed adequate time for repair and recovery so as to ensure reasonable reconstruction of alveolar bone and healthy movement of the orthodontic tooth.

In conclusion, these findings indicated that on day 14 , the rats of 25-g group that were in repair and reconstruction phase were feasible to apply continuous force load, however, it was disputable to continue applying any force loading for the rats of 75-g group.

In addition, the movement distance in 75-g group was significantly larger only at day 14, compared with 25-g group. Apparently, the fast tooth movement at the expense of excessive alveolar bone resorption was not ideal.

Taken together, frequently applying orthodontic force loading during orthodontic treatment was demonstrated to be unhelpful. In order to maintain the health of periodontal tissues, adequate time for repair and recovery is needed to ensure reasonable remolding of alveolar bone and healthy movement of the orthodontic tooth.

\section{Acknowledgements}

This study was supported by the National Natural Science Foundation of China (grant no. 81170960) and the Special Foundation for Sino-Russian Translational Medicine Research Center of Harbin Medical University (grant no. CR201412).

\section{References}

1. Henneman S, Von den Hoff JW and Maltha JC: Mechanobiology of tooth movement. Eur J Orthod 30: 299-306, 2008.

2. Wise GE and King GJ: Mechanisms of tooth eruption and orthodontic tooth movement. J Dent Res 87: 414-434, 2008.

3. Salazar M, Hernandes L, Ramos AL, Micheletti KR, Albino CC and Nakamura Cuman RK: Effect of teriparatide on induced tooth displacement in ovariectomized rats: A histomorphometric analysis. Am J Orthod Dentofacial Orthop 139: e337-e344, 2011.

4. Martin-Badosa E, Amblard D, Nuzzo S, Elmoutaouakkil A, Vico L and Peyrin F: Excised bone structures in mice: imaging at three-dimensional synchrotron radiation micro $\mathrm{CT}$. Radiology 229: 921-928, 2003.

5. Waarsing JH, Day JS, van der Linden JC, Ederveen AG, Spanjers C, De Clerck N, Sasov A, Verhaar JA and Weinans H: Detecting and tracking local changes in the tibiae of individual rats: A novel method to analyse longitudinal in vivo micro-CT data. Bone 34: 163-169, 2004

6. Dempster DW, Compston JE, Drezner MK, Glorieux FH, Kanis JA, Malluche H, Meunier PJ, Ott SM, Recker RR and Parfitt AM: Standardized nomenclature, symbols, and units for bone histomorphometry: a 2012 update of the report of the ASBMR Histomorphometry Nomenclature Committee. J Bone Miner Res 28: 2-17, 2013.

7. Parfitt AM, Mathews CH, Villanueva AR, Kleerekoper M, Frame B and Rao DS: Relationships between surface, volume, and thickness of iliac trabecular bone in aging and in osteoporosis. Implications for the microanatomic and cellular mechanisms of bone loss. J Clin Invest 72: 1396-1409, 1983.

8. Waldo CM and Rothblatt JM: Histologic response to tooth movement in the laboratory rat; procedure and preliminary observations. J Dent Res 33: 481-486, 1954.

9. Ren Y, Maltha JC and Kuijpers-Jagtman AM: The rat as a model for orthodontic tooth movement - a critical review and a proposed solution. Eur J Orthod 26: 483-490, 2004.

10. Gonzales C, Hotokezaka H, Arai Y, Ninomiya T, Tominaga J, Jang I, Hotokezaka Y, Tanaka M and Yoshida N: An in vivo 3D micro-CT evaluation of tooth movement after the application of different force magnitudes in rat molar. Angle Orthod 79: 703-714, 2009

11. Xu Y, Zhao T, Xu W and Ding Y: Periodontal microstructure change and tooth movement pattern under different force magnitudes in ovariectomized rats: An in-vivo microcomputed tomography study. Am J Orthod Dentofacial Orthop 143: 828-836, 2013.

12. Xu X, Zhou J, Yang F, Wei S and Dai H: Using micro-computed tomography to evaluate the dynamics of orthodontically induced root resorption repair in a rat model. PLoS One 11: e0150135, 2016.

13. Kohno T, Matsumoto Y, Kanno Z, Warita H and Soma K: Experimental tooth movement under light orthodontic forces: rates of tooth movement and changes of the periodontium. J Orthod 29: 129-135, 2002.

14. Tomizuka R, Shimizu Y, Kanetaka H, Suzuki A, Urayama S, Kikuchi M, Mitani H and Igarashi K: Histological evaluation of the effects of initially light and gradually increasing force on orthodontic tooth movement. Angle Orthod 77: 410-416, 2007.

15. Reitan K and Kvam E: Comparative behavior of human and animal tissue during experimental tooth movement. Angle Orthod 41: 1-14, 1971 .

16. Storey E: The nature of tooth movement. Am J Orthod 63: 292-314, 1973.

17. King GJ, Keeling SD, McCoy EA and Ward TH: Measuring dental drift and orthodontic tooth movement in response to various initial forces in adult rats. Am J Orthod Dentofacial Orthop 99: 456-465, 1991. 